\title{
Elastic constants and high-pressure structural transitions in lanthanum monochalcogenides from experiment and theory
}

\author{
G. Vaitheeswaran ${ }^{1, *}$, V. Kanchana ${ }^{1}$, S. Heathman ${ }^{2}$, M. Idiri², \\ T. Le Bihan ${ }^{3}$, A. Svane ${ }^{4}$, A. Delin ${ }^{1}$ and B. Johansson ${ }^{1,5}$ \\ ${ }^{1}$ Applied Materials Physics, Department of Materials Science and Engineering, \\ Royal Institute of Technology, Brinellvägen 23, 10044 Stockholm, Sweden \\ ${ }^{2}$ European Commission, JRC, Institute for Transuranium Elements, \\ Postfach 2340, D-76125, Karlsruhe, Germany \\ ${ }^{3}$ European Synchrotron Radiation Facility, \\ BP220, F-38043 Grenoble Cedex, France \\ ${ }^{4}$ Department of Physics and Astronomy, \\ University of Aarhus, DK-8000 Aarhus C, Denmark \\ ${ }^{5}$ Condensed Matter Theory Group, Department of Physics, \\ Uppsala University, Box.530, SE-751 21, Uppsala, Sweden
}

(Dated: September 20, 2018)

\begin{abstract}
The high-pressure structural behavior of lanthanum monochalcogenides is investigated by theory and experiment. Theory comprises density functional calculations of LaS, LaSe and LaTe with the general gradient approximation for exchange and correlation effects, as implemented within the full-potential linear muffin-tin orbital method. The experimental studies consist of high-pressure angle dispersive x-ray diffraction investigations of LaS and LaSe up to a maximum pressure of 41 GPa. A structural phase transition from the $\mathrm{NaCl}$ type to $\mathrm{CsCl}$ type crystal structure is found to occur in all cases. The experimental transition pressures are 27-28 GPa and 19 GPa, for LaS and LaSe, respectively, while the calculated transition pressures are $29 \mathrm{GPa}, 21 \mathrm{GPa}$ and $10 \mathrm{GPa}$ for LaS, LaSe and LaTe, respectively. The calculated ground state properties such as equilibrium lattice constant, bulk modulus and its pressure derivative, and Debye temperatures are in good agreement with experimental results. Elastic constants are predicted from the calculations.
\end{abstract}




\section{INTRODUCTION}

The lanthanum monochalcogenides belong to the wide class of binary rare-earth monochalcogenides with the NaCl-type structure, which has been intensively studied because of their interesting physical properties including complex magnetic structures, pressure induced insulator-metal transitions, anomalous valence fluctuations, and unusual Fermi surface properties. 1,2 The trivalent lanthanum monochalcogenides are superconductors near $1 \mathrm{~K}$, and the superconducting transition temperature as well as the electronic specific heat coefficient increases from the monosulfide to the monotelluride, whereas the Debye temperature decreases from LaS to $\mathrm{LaTe}^{3}$.

In this work we explore the high pressure behavior of the La chalcogenides by experiment and theory. High-pressure x-ray diffraction experiments are conducted on LaS and LaSe to obtain equations of states including discontinuous structural transitions from the $\mathrm{NaCl}$ structure (Space group Fm3m, Z=1, also called the B1 structure in the following) to the $\mathrm{CsCl}$ structure (Space group Pm3m, Z=4, also termed the B2 structure in the following). Density functional calculations are performed to compare basic theoretical predictions with the measurements. The present experimental study of LaS extends our previous study on $\mathrm{U}_{x} \mathrm{La}_{1-x} \mathrm{~S}$ compounds ${ }^{4}$. For LaSe no high-pressure results have been reported previously, while high-pressure experiments on LaTe report a structural transition from B1 to B2 at around $7 \mathrm{GPa}^{5}$.

A second objective of the present work is to investigate the elastic properties of the lanthanum chalcogenides, for which there are no experimental results available. Elastic constants are derived from total energy variations with applied strains. We compare our results with recent theoretical work ${ }^{6}$ and find good agreement.

The systematics of the electronic band structure through the lanthanum and cerium chalcogenide series was studied with angle-resolved photoemission spectroscopy $\underline{7}^{7}, \underline{8}$. The Fermi surface properties of lanthanum monochalcogenides have been studied by de Haas-van Alphen effect measurements $\underline{9}, 10$. From the theoretical side the energy band structure, superconductivity, surface electronic structure, optical and magneto-optical spectra as well as structural stability of lanthanum monochalcogenides have been investigated by several authors using the local spin density approximation (LSDA) as well as the LSDA+U approximation $\underline{11}, 12,13,14,15,16,17$. A few papers also focused on the calculation of second and 
third order elastic constants using the short range repulsive potential method $\underline{18}, \underline{19}, 20$. A few experimental studies such as point contact spectroscopy ${ }^{21}$, reflectivity ${ }^{22}$ and phonon spectra $^{23}$ have been reported for LaS. The magneto-optical properties of lanthanum chalcogenides have been investigated experimentally, $24,25,26$ where a non-zero Kerr effect could be observed in these Pauli paramagnets by applying an external magnetic field.

The rest of the paper is organized as follows. In section 2 we discuss the computational and experimental details of our work. The calculated ground state properties, elastic constants as well as the experimental and theoretical results for the high-pressure behavior and structural transitions are presented in Section 3, and the conclusions are given in Section 4.

\section{COMPUTATIONAL AND EXPERIMENTAL DETAILS}

\section{A. The electronic structure method}

In this work we have used the all-electron full-potential linear muffin tin orbital (FPLMTO) $\operatorname{method}^{27}$ to calculate the total energies and the basic ground state properties. Here the crystal is divided into two regions: non-overlapping muffin-tin spheres surrounding each atom and the interstitial region between the spheres. We used a double $\kappa$ spdf LMTO basis (each radial function within the spheres is matched to a Hankel function in the interstitial region) for describing the valence bands. The following basis set was used in the calculations: $\mathrm{La}(5 \mathrm{~s}, 6 \mathrm{~s}, 5 \mathrm{p}, 5 \mathrm{~d}, 4 \mathrm{f}), \mathrm{S}(3 \mathrm{~s}, 3 \mathrm{p}, 3 \mathrm{~d}), \mathrm{Se}(4 \mathrm{~s}, 4 \mathrm{p}, 3 \mathrm{~d}, 4 \mathrm{~d}), \mathrm{Te}(5 \mathrm{~s}, 5 \mathrm{p}, 4 \mathrm{~d}, 5 \mathrm{~d})$. The exchange correlation potential was calculated within the generalized gradient approximation (GGA) scheme ${ }^{28}$. The charge density and potential inside the muffin-tin spheres were expanded in terms of spherical harmonics up to $l_{\max }=6$, while in the interstitial region, they were expanded in plane waves, with 6566 (energy up to $109.80 \mathrm{Ry}$ ) waves being included in the calculation. Total energies were calculated as a function of volume, for a (18 18 18) k-mesh, corresponding to $195 \mathrm{k}$-vectors in the irreducible wedge of the Brillouin zone, and the results fitted to the Birch equation of state ${ }^{29}$ to obtain the ground state properties.

The elastic constants were obtained from the variation of the total energy under volumeconserving strains, as outlined in Ref. 30. 


\section{B. Experimental details}

Experiments were carried out at the European Synchrotron Radiation Facility (ESRF), on the ID30 beamline dedicated to high pressure diffraction experiments, in the angular dispersive mode, using a double focused monochromatic beam at $\lambda=0.3738 \AA$. Loading of LaS and LaSe powders were performed in Le Toullec-type diamond anvil cells (DAC), using nitrogen, argon or silicone oil as the pressure transmitting media. The pressure inside the cell was determined via the ruby fluorescence method ${ }^{31}$ from a small ruby ball mounted alongside the LaX sample. The sample to detector distance was calibrated before each set of experiments by means of a standard silicon powder sample. Diffraction images were captured with a FASTSCAN32 image plate detector, and processed using the ESRF Fit2D program $^{33}$ to provide data in a format suitable for Rietveld analysis using the FULLPROF program ${ }^{34}$. The lattice constants at ambient pressure have been determined $\mathrm{as}_{0}=5.852$ and $\mathrm{a}_{0}=6.067 \AA$ for LaS and LaSe, respectively, in good agreement with previous experimental data given by Ref. 16.

\section{STRUCTURAL, ELASTIC AND HIGH PRESSURE STUDIES}

\section{A. Ground state and Elastic properties}

The calculated ground state properties such as equilibrium lattice constant, bulk modulus and its pressure derivative are given in Table 1. The calculated equilibrium lattice constants are generally overestimated by $\sim 0.5 \%$ compared to experiment, which is an improvement compared to earlier calculations $\frac{12,16}{}$. Similarly, for the bulk modulus, the agreement between theory and experiment has improved $\underline{12}, \underline{16}$. It is interesting to note that the bulk moduli of the lanthanum chalcogenides are quite similar to those of the neighboring cerium chalcogenides, which are characterized by a localized $f$ state $^{37}: B=82 \mathrm{GPa}, 76 \mathrm{GPa}$ and $58 \mathrm{GPa}$, for CeS, CeSe and CeTe, respectively. Similarly, the calculated bulk moduli of the praseodymium chalcogenides are $B=89 \mathrm{GPa}, 78 \mathrm{GPa}$ and $57 \mathrm{GPa}$, for PrS, PrSe and PrTe, respectively, when $\operatorname{Pr}$ is represented in a trivalent configuration ${ }^{38}$. These similarities of numbers corroborate the practice of taking the lanthanum chalcogenides as non-magnetic reference systems for the later rare-earth chalcogenides.

The calculated elastic constants of LaS, LaSe and LaTe are listed in Table 2, where we also 
compare to the recent LAPW calculation of Ref. 6. The two theoretical approaches find very similar $C_{11}$ value for LaS and LaSe, while for LaTe this parameter is $\sim 13 \%$ smaller in the present calculations than found by the LAPW method. The present $C_{12}$ and $C_{44}$ parameters are in all cases lower than found in Ref. 6, for LaSe $C_{12}$ with almost a factor of 2 difference. Our calculated shear moduli $G$ are $19 \%$ to $40 \%$ larger than the ones calculated with LAPW. For Young's modulus E, our values are 16\% to $35 \%$ higher, and finally, for Poisson's ratio $\nu$ our values are $8 \%$ to $17 \%$ lower than the LAPW results. The largest discrepancies between the two calculations occur for LaTe in all cases. Furthermore, we observe the general trend that the heavier the chalcogenide is, the softer is the compound. This trend is expected and easy to understand, since with increased chalcogen size, the lattice parameter increases and the valence orbitals become increasingly delocalized due to a higher number of nodes $\underline{39}$

As previously stated, there is at present no experimental information regarding the elastic constants available for the lanthanum chalcogenides. The elastic constants of LaS are of roughly the same magnitude as those measured for the isoelectronic YS compound (Table 2).

Table 3 presents sound velocities and Debye temperatures, as derived from the calculated elastic constants ${ }^{30}$. The Debye temperatures have been determined experimentally from the low-temperature specific heat, and the agreement with our calculations is excellent (the calculated Debye temperatures are $0.5 \%$ to $6 \%$ higher than experiment), which can be taken as an indirect check on our calculated elastic constant values.

\section{B. High-pressure structural transitions}

The high pressure structural behavior of the lanthanum chalcogenides was studied both experimentally and theoretically. The calculated total energies as functions of relative volume for LaS, LaSe and LaTe are shown in figure 1, while experimental and theoretical pressure-volume relations are presented in figures 2, 4 and 5 for LaS, LaSe and LaTe, respectively. Figure 3 shows the diffraction spectra recorded for LaS and LaSe. It appears from the theory presented in figure 1 that with compression the B2 phase becomes more and more favorable, and eventually a transition from the B1 structure to the B2 structure occurs. From the common tangent of the B1 and B2 total energies the transition pressure is determined. The calculated transition pressure for LaS is $29.3 \mathrm{GPa}$ with a volume collapse 
of $10.3 \%$, which is in good agreement with the experimental transition pressure of 27-28 GPa with a volume collapse of around 9.5\% as shown in figure 2. A similar transition was predicted to occur in $\mathrm{CeS}$ around $24.3 \mathrm{GPa}$, in which the Ce ions are in a tetravalent state 41 In the case of $\mathrm{PrS}$ a similar transition is predicted to occur around $22 \mathrm{GPa}$, in this case with the $\operatorname{Pr}$ ions remaining trivalent $\frac{38}{}$. The transition pressure of LaS is similar to that of the pnictogen group neighbour LaP, in which a transition from the B1 structure to a distorted B2 structure is observed experimentally around $24 \mathrm{GPa}^{42}$.

Like LaS, LaSe also undergoes a B1 $\rightarrow$ B2 transition, experimentally seen at a pressure of $19 \mathrm{GPa}$ with a volume reduction of 10\%, cf. figure 4, for which the present theory finds concordant values of $P_{t}=21 \mathrm{GPa}$ with a volume collapse of $10.4 \%$. In the case of CeSe a similar transition from B1 type to B2 type occurs around $20 \mathrm{GPa} 37$, in this case with the Ce ion remaining trivalent on both sides of the transition according to theory $\stackrel{41}{ }$. Even for PrSe a B1 $\rightarrow$ B2 transition is predicted to occur around $12 \mathrm{GPa}$, wherein the Pr ions remain trivalent ${ }^{38}$. The transition pressure of LaSe is similar to that of the pnictogen neighbour LaAs, in which a transition from B1 structure to a distorted B2 type occurs around 20 $\mathrm{GPa}^{43}$.

As far as LaTe is concerned a transition from B1 type to B2 type is reported around $7 \mathrm{GPa}^{5}$, which agrees quite well with the calculated transition pressure of $9.7 \mathrm{GPa}$ with a volume reduction of $10.4 \%$, cf. figure 5. Both CeTe and PrTe undergo a transition from B1 type to $\mathrm{B} 2$ around $8 \pm 1 \mathrm{GPa}^{44}$ and $9 \pm 1 \mathrm{GPa}^{45}$. When comparing the pnictogen neighbour LaSb with that of LaTe the pnitogen undergoes a transition from B1 type to a distorted B2 type structure around $11 \mathrm{GPa}^{46}$ which is slightly higher than that of LaTe.

\section{CONCLUSIONS}

By means of a combined theoretical and experimental study the high-pressure structural behavior of $\operatorname{LaX}(\mathrm{X}=\mathrm{S}$, Se, Te) compounds have been investigated. Unlike the lanthanum monopnictides which show a transition from B1 to a distorted B2 structure ${ }^{47}$, 48 , the lanthanum monochalcogenides exhibit a simple B1 $\rightarrow$ B2 structural phase transition, similar to the one found for most of the lanthanide and actinide monochalcogenides studied up to now $2,49,50,51,52,53$.

For all three systems studied here, the volume collapse is around 10\% (from both experi- 
ment and theory) at the B1 $\rightarrow$ B2 transition. Let us compare this magnitude of the volume collapse to a very simple model in which we assume that at the transition point, the La-X bond length is restored to the ambient value. We expect such a model to underestimate the volume collapse, since the bond length at high pressure most probably will be a bit smaller than at ambient pressure. Our simple model gives the following relation between the relative volume $V_{t}^{B 1} / V_{0}^{B 1}$ just before the transition and the volume collapse $1-V_{t}^{B 2} / V_{t}^{B 1}$ :

$$
1-\frac{V_{t}^{B 2}}{V_{t}^{B 1}}=1-\frac{4}{3 \sqrt{3}} \frac{V_{0}^{B 1}}{V_{t}^{B 1}} .
$$

For LaS, the experimental relative volume at the transition is 0.82 , which would give a volume collapse of $6 \%$ according to this model. For LaSe, the corresponding numbers are 0.84 and $8 \%$. Thus, the volume collapse observed experimentally (and from density functional calculations) can be viewed as a partial restoration of the original bond length between the La and the chalcogenide. Based on the above discussion we conclude that the volume collapse observed in the lanthanum chalcogenides is entirely consistent with a simple picture of the transition in which the volume collapse is a consequence of the rearrangement of the atoms to a more close-packed structure while the total number of valence electrons remains unchanged. We have also checked the partial occupation number of spdf states across the structural transition and there is no appreciable change in the occupation numbers across the transition. This is in contrast to the situation in many Ce systems, in which the volume collapse is accompanied by delocalization of an $f$ electron. $11,54,55,56$

\section{Acknowledgments}

G. V, V. K, A. D, and B. J acknowledge V. R. and SSF for the financial support and SNIC for the computer time. The authors S.H. , M.I. and T.L.B. would like to thank K. Mattenberger and O. Vogt (ETH, Zürich) for the samples used in this study. Support given to M.I. within the framework of the EC funded program "Human capital and mobility" is also acknowledged.

* Author for Correspondence, E-mail: vaithee@kth.se

1 A. Jayaraman, P. D. Dernier, and L. D. Longinotti, High Temp. High Press. 7, 1 (1975). 
2 U. Benedict, J. Alloys Comp. 223, 216 (1995).

3 E. Bucher, K. Andres, F. J. di Salvo, J. P. Maita, A. C. Gossard, A. S. Cooper and G. W. Hull, Jr., Phys. Rev. B 11, 500 (1975).

4 T. Le Bihan, A. Bombardi, M. Idiri, S. Heathman and A. Lindbaum, J. Phys.: Condens. Matter 14, 10595 (2002).

5 A. Jayaraman, Bull. Amer. Phys. Soc. 24, 397 (1978).

6 A. Bouhemadou, R. Khenata, and M. Maamache, J. Mol. Struc.: THEOCHEM (2006), doi:10.1016/j.theochem.2006.08.031

7 M. Nakayama, T. Ito, H. Kumigashira, H. Matsui, H. Komatsu, T. Takahashi, H. Aoki and A. Ochiai, J. Magn. Magn. Mater. 272-276, e121 (2004).

8 M. Nakayama, H. Aoki, A. Ochiai, T. Ito, H. Kumigashira, T. Takahashi and H. Harima, Phys. Rev. B 69, 155116 (2004).

9 M. Nakayama, N. Kimura, H. Aoki, C. Terakura, T. Terashima, S. Uji and A. Ochiai, J. Magn. Magn. Mater. 272-276, e93 (2004).

10 M. Nakayama, N. Kimura, H. Aoki, A. Ochiai, C. Terakura, T. Terashima and S. Uji, Phy. Rev. B 70, 054421 (2004).

11 S. V. Vlasov and O. V. Farberovich, Solid State Commun. 56, 967 (1985).

12 Z. W. Lu, D. J. Singh and H. Krakauer, Phys. Rev. B 37, 10045 (1988).

13 S. Sankaralingam, S. M. Jaya, G. Pari and R. Asokamani, Phys. Stat. Solidi (b) 174, 435 (1992).

14 O. Eriksson, J. Wills, P. Mumford, M. Cahay and W. Friz, Phys. Rev. B 57, 4067 (1998).

15 O. Eriksson, M. Cahay and J. M. Wills, Phys. Rev. B 65, 033304 (2001).

16 G. Vaitheeswaran, V. Kanchana and M. Rajagopalan, J. Phys. Chem. Solids 64, 15 (2003).

17 V. N. Antonov, B. N. Harmon and A. N. Yaresko, Phys. Rev. B 69, 094404 (2004).

18 R. R. Yadav and D. Singh, J. Phys. Soc. Japan 70, 1825 (2001).

19 D. Varshney, N. Kaurav, P. Sharma, S. Shah and R. K. Singh, Phys. Stat. Solidi (b) 241, 3179 (2004).

20 D. Varshney, N. Kaurav, R. Kinge, S. Shah and R. K. Singh, High Press. Res. 25, 145 (2005).

21 I. Frankowski and P. Wachter, Solid State Commun. 40, 885 (1985).

22 W. Beckenbaugh, J. Evers, G. Güntherodt, E. Kaldis and P. Wachter, J. Phys. Chem. Solids 36, 239 (1975).

23 M. M. Steiner, H. Eschrig and R. Monnier, Phys. Rev. B 45, 7183 (1992). 
29 F. Birch, J. Appl. Phys. 9, 279, (1938).

30 V. Kanchana, G. Vaitheeswaran, A. Svane and A. Delin, J. Phys.: Condens. Matter, 18, 9615 (2006).

31 H. K. Mao, J. Xu and P. M. Bell, J. Geophys. Res. 91, 4673, (1986).

32 M. Thoms, S. Bauchau, D. Haüsermann, M. Kuntz, T. Le Bihan, M. Mezouar and D. Strawbridge, Nucl. Instrum. Methods Phys. Res. A 413, 175 (1998).

33 A. P. Hammersley, S. O. Svensson, M. Hanfland, A. N. Fitch and D. Haüsermann, High Press. Res. 14, 235 (1996).

34 J. Rodriguez-Carvajal, Physica B 192, 55 (1993).

35 S. Von Molnar, T. Penny and F. Holtzberg, J. De Physique 37, C4-241 (1976).

36 A. Jayaraman, B. Batlogg, R. G. Maines and H. Bach Phys. Rev. B 26, 3347 (1982).

37 J. M. Léger, Physica B 190, 84 (1993).

38 G. Vaitheeswaran, L. Petit, A. Svane, V. Kanchana and M. Rajagopalan, J. Phys.: Condens. Matter 16, 4429 (2004).

39 A. Delin, P. Ravindran, O. Eriksson, and J. M. Wills, Int. J. Quantum Chem. 69, 349 (1998);

A. Delin and T. Klüner, Phys. Rev. B 66, 035117 (2002).

40 P. D. Dernier, W. Weber and L. D. Longinotti, Phys. Rev. B 14, 3635 (1976).

41 A. Svane, W. Temmerman and Z. Szotek, Phys. Rev. B 59, 7888 (1999).

42 T. Adachi, I. Shirotani, J. Hayashi and O. Shimomura, Phys. Lett. A 250, 389 (1998).

43 I. Shirotani, K. Yamanashi, J. Hayashi, N. Ishimatsu, O. Shimomura, and T. Kikegawa, Solid State Communications 127, 573 (2003).

44 J. M. Léger, R. Epain, J. Loriers, D. Ravot, J. Rossat-Mignod, Phys. Rev. B 28, 7125 (1983).

45 A. Chatterjee, A. K. Singh, and A. Jayaraman, Phys. Rev. B 6, 2285 (1972).

46 J. Hayashi, I. Shirotani, Y. Tanaka, T. Adachi, O. Shimomura, and T. Kikegawa, Solid State Commun. 114, 561 (2000).

47 G. Vaitheeswaran, V. Kanchana and M. Rajagopalan, J. Alloys Comp. 336, 46 (2002). 
48 G. Vaitheeswaran, V. Kanchana and M. Rajagopalan, Physica B 315, 64 (2002).

49 S. Dabos-Seignon, U. Benedict, S. Heathman, J. C. Spirlet and M. Pages, J. Less-Common Met. 160, 35 (1990).

50 M. Gensini, E. Gering, S. Heathman, U. Benedict and J. C. Spirlet, High Press. Res. 2, 347 (1990).

51 L. Gerward, J. S. Olsen, U. Benedict, S. Dabos and O. Vogt, High Press. Res. 1, 235 (1989).

52 T. Le Bihan, S. Darracq, S. Heathman, U. Benedict, K. Mattenberger and O. Vogt, J. Alloys Comp. 226, 143 (1995).

53 T. Le Bihan, S. Heathman and J. Rebizant High Press. Res. 15, 387 (1997).

54 B. Johansson, Phil. Mag. 30, 469 (1974).

55 A. Delin, P. M. Oppeneer, M. S. S. Brooks, T. Kraft, J. M. Wills, B. Johansson, and O. Eriksson, Phys. Rev. B 55 R10173 (1997).

56 A. Svane, Z. Szotek, W. M. Temmerman and H. Winter, Solid State Commun. 102, 473 (1997). 
TABLE I: Calculated lattice constants in $\AA$, bulk modulus in GPa, and its pressure derivative $B^{\prime}$ of the lanthanum monochalcogenides in the B1 structure

\begin{tabular}{ccccc}
\hline \hline Compound & & Lattice Constant & Bulk Modulus & $B^{\prime}$ \\
\hline \multirow{2}{*}{ LaS } & Expt. this work & 5.852 & $89(3), 83.6^{d}$ & $6.5(4)$ \\
& Theory, this work & 5.873 & 87.8 & 3.95 \\
& Other theory & $5.812^{a}, 5.773^{b}, 5.727^{c}, 5.895^{e}$ & $97.74^{a}, 107^{c}, 81.53^{e}$ & $4.67^{e}$ \\
LaSe & Expt. this work & 6.067 & $74(2)$ & $4.7(3)$ \\
& Theory, this work & 6.091 & 74.8 & 4.12 \\
& Other theory & $5.957^{c}, 6.126^{e}$ & $97.74^{c}, 68.40^{e}$ & $4.28^{e}$ \\
LaTe & Expt. & $6.435^{h}$ & $60.6 \pm 2^{f}, 55^{g}$ & - \\
& Theory, this work & 6.470 & 59.4 & 4.12 \\
& Other theory & $6.255^{c}, 6.512^{e}$ & $74.02^{c}, 55.34^{e}$ & $4.96^{e}$ \\
\hline \hline
\end{tabular}

${ }^{a} \operatorname{Ref} .12 ;{ }^{b} \operatorname{Ref} .14 ;{ }^{c} \operatorname{Ref} .16 ;{ }^{d} \operatorname{Ref} .35 ;{ }^{e} \operatorname{Ref} .6 ;{ }^{f} \operatorname{Ref} .36 ;{ }^{g} \operatorname{Ref.} .2 ;{ }^{h} \operatorname{Ref} .10$.

TABLE II: Calculated elastic constants, shear modulus $G$, Young's modulus $E$ - all given in GPa - and Poisson's ratio $\nu$ for lanthanum monochalcogenides in the B1 structure at the theoretical equilibrium volume. For comparison, results of LAPW calculations (Ref. 66) and experimental results for YS (Ref. 40) are included.

\begin{tabular}{|c|c|c|c|c|c|c|c|}
\hline Compound & $C_{11}$ & $C_{12}$ & $C_{44}$ & $\mathrm{G}$ & $\mathrm{E}$ & $\nu$ & \\
\hline \multirow[t]{2}{*}{$\mathrm{LaS}$} & 227.9 & 18.0 & 22.2 & 55.3 & 137.2 & 0.240 & Present \\
\hline & 234 & 23 & 25 & 46.6 & 117.8 & 0.26 & LAPW \\
\hline YS & 250 & 20 & 30 & - & - & - & Expt. \\
\hline \multirow[t]{2}{*}{ LaSe } & 201.6 & 11.4 & 15.7 & 47.5 & 117.5 & 0.238 & Present \\
\hline & 203 & 21 & 22 & 40.6 & 102.5 & 0.26 & LAPW \\
\hline \multirow[t]{2}{*}{ LaTe } & 158.7 & 9.7 & 7.9 & 34.5 & 86.8 & 0.256 & Present \\
\hline & 171 & 12 & 8 & 24.6 & 64.1 & 0.30 & LAPW \\
\hline
\end{tabular}


TABLE III: Calculated longitudinal, shear, and average wave velocity $\left(v_{l}, v_{s}\right.$, and $v_{m}$, respectively) in $\mathrm{m} / \mathrm{s}$, and the Debye temperature, $\theta_{D}$, in kelvin from the average elastic wave velocity for lanthanum monochalcogenides in the B1 structure at the theoretical equilibrium volume.

\begin{tabular}{cccccc}
\hline \hline Compound & & $v_{l}$ & $v_{s}$ & $v_{m}$ & $\theta_{D}$ \\
\hline LaS & Present & 5249 & 3072 & 3406 & 277.2 \\
& Expt. $^{a}$ & - & - & - & 276 \\
LaSe & Present $^{*}$ & 4626 & 2712 & 3006 & 233.6 \\
& Expt. $^{a}$ & - & - & - & 231 \\
LaTe & Present $^{*}$ & 3972 & 2273 & 2524 & 185.7 \\
& Expt. $^{a}$ & - & - & - & 175 \\
\hline \hline
\end{tabular}

${ }^{a} \operatorname{Ref}, \overline{3}$

TABLE IV: Calculated and experimental transition pressures, $P_{t}$ in GPa, and volume changes, given in $\%$, for the B1 $\rightarrow$ B2 structural phase transition of lanthanum monochalcogenides.

\begin{tabular}{ccccc}
\hline \hline Compound & & $P_{t}$ & \multicolumn{2}{c}{ Volume Collapse } \\
& Theory & Expt. & Theory & Expt. \\
\hline LaS & 29.3 & $27-28$ & 10.3 & 9.5 \\
LaSe & 21 & 19 & 10.4 & 10.5 \\
LaTe & 9.7 & $7^{a}$ & 10.4 & - \\
\hline \hline
\end{tabular}



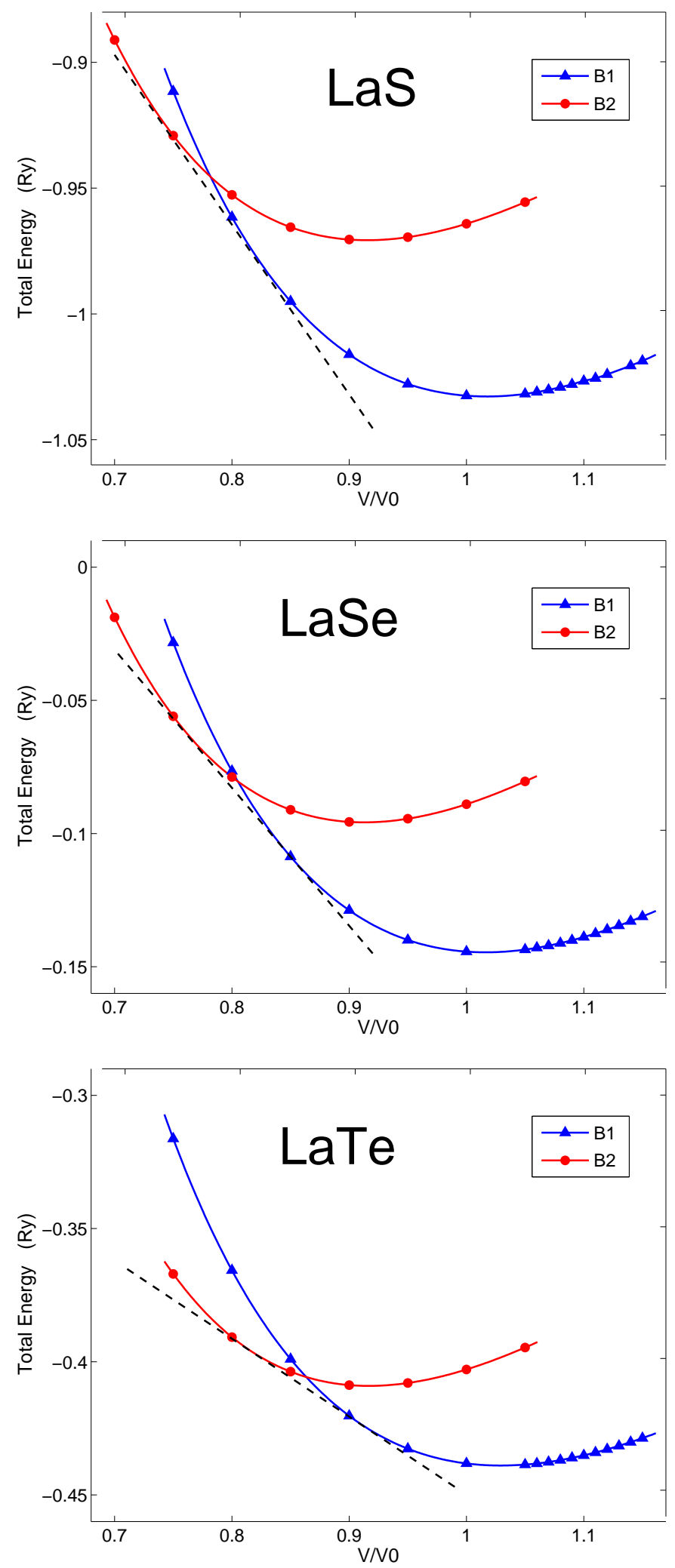

FIG. 1: (Color online) Calculated total energy vs. relative volume for (a): LaS, (b): LaSe, and (c): LaTe in the B1 and B2 structures. The common tangents mark the structural B1 $\rightarrow$ B2 transition. 


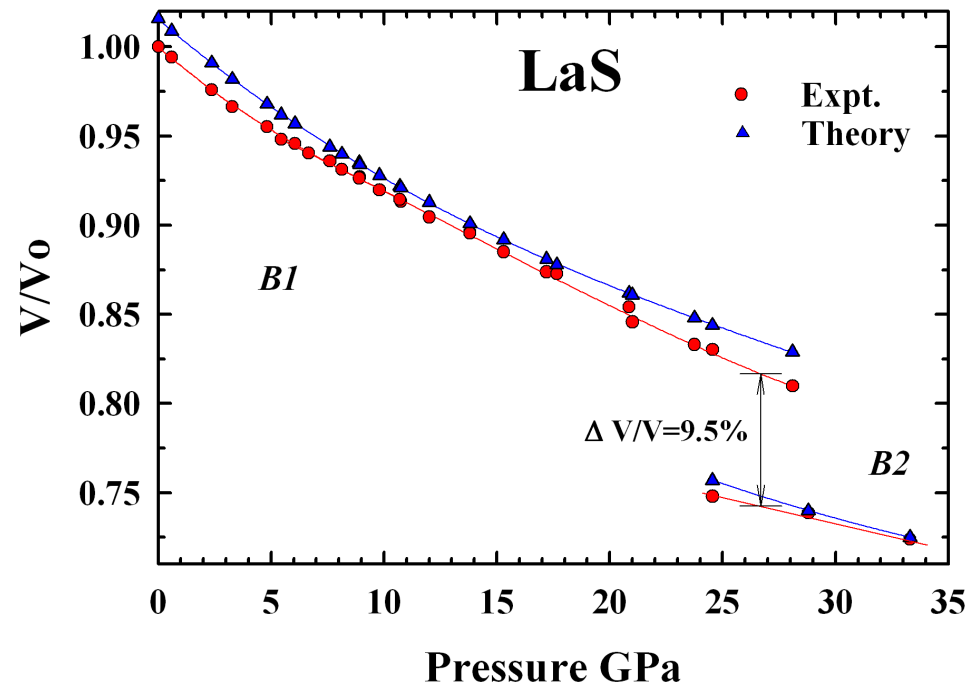

FIG. 2: (Color online) Pressure as a function of relative volume for LaS in the B1 and B2 structures. Triangles: theory (GGA), circles: experiment. The experimental volume collapse at the B1 $\rightarrow$ B2 transition is marked. 

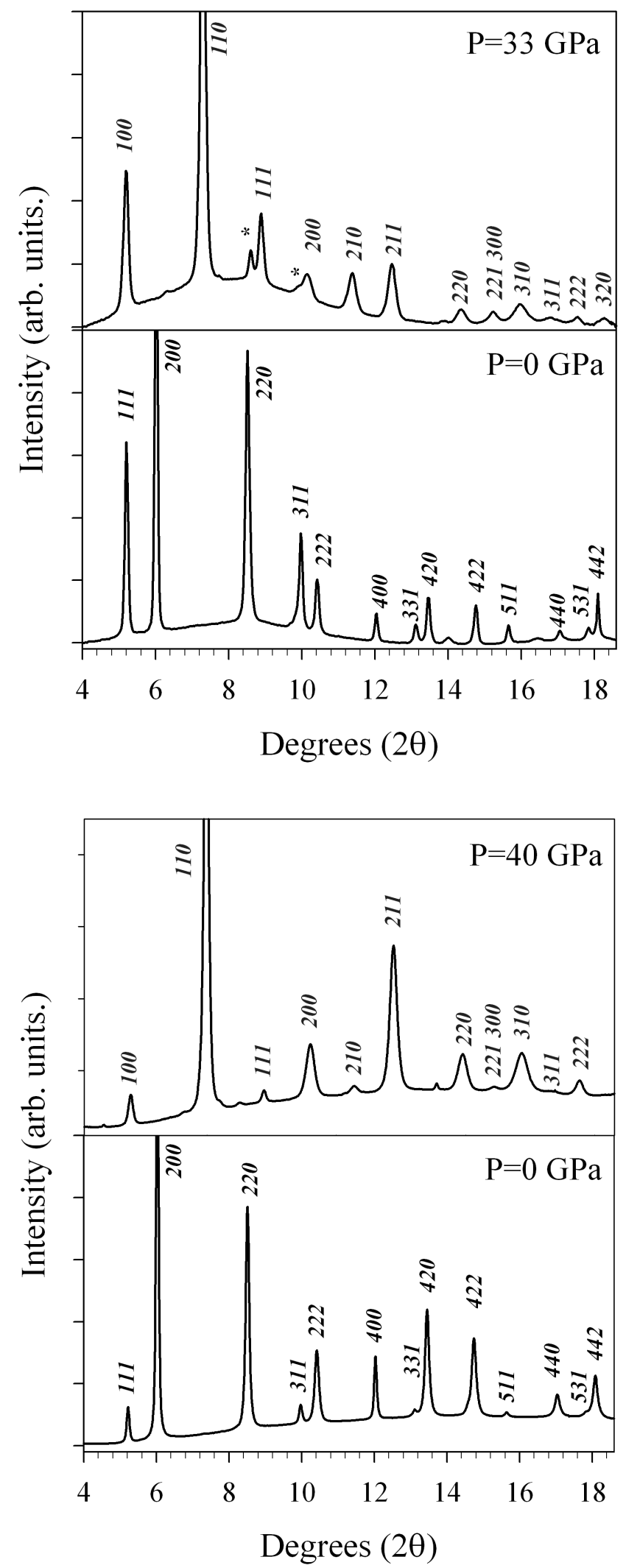

FIG. 3: X-ray diffraction spectra of (a): LaS and (b): LaSe in the B1 structure at $P=0 \mathrm{GPa}$, $a=5.8520 \AA$ for LaS, $a=6.0670 \AA$ for LaSe, and in the B2 structure right after the transition, at 


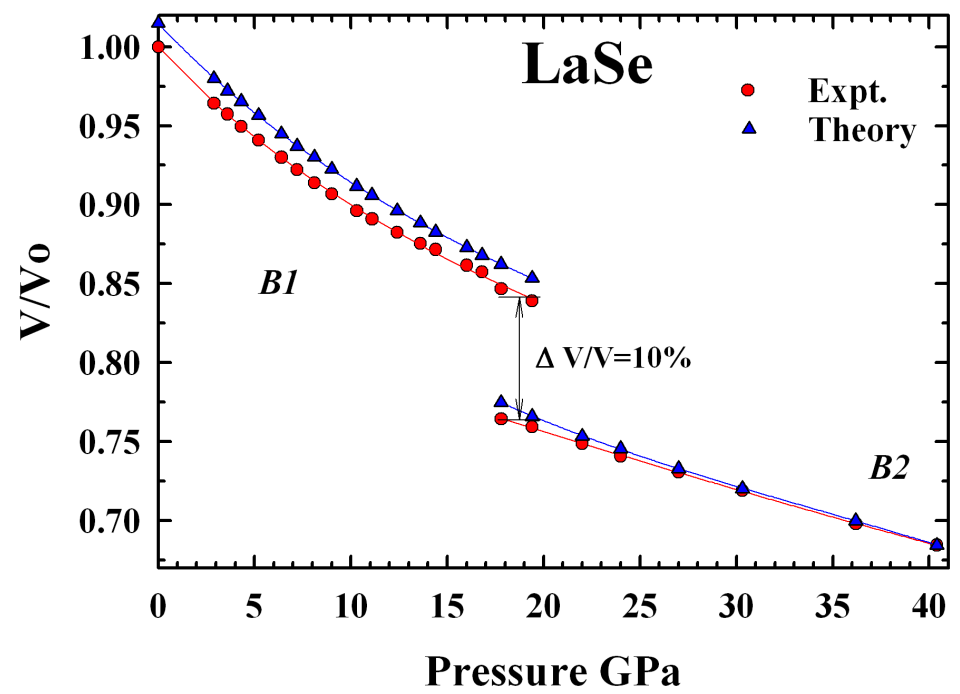

FIG. 4: (Color online) Pressure as a function of relative volume for LaSe in the B1 and B2 structures. Triangles: theory (GGA), circles: experiment. The experimental volume collapse at the $\mathrm{B} 1 \rightarrow \mathrm{B} 2$ transition is marked.

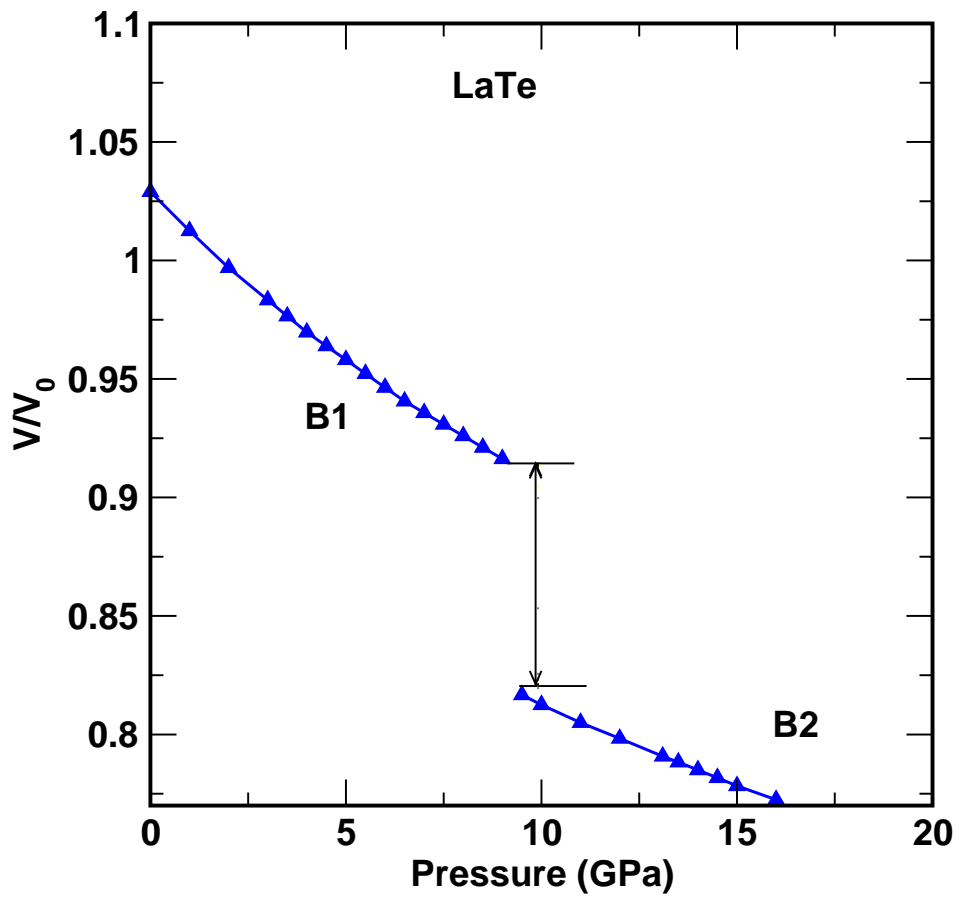

FIG. 5: (Color online) Theoretical pressure as a function of relative volume for LaTe in the B1 and B2 structures. 